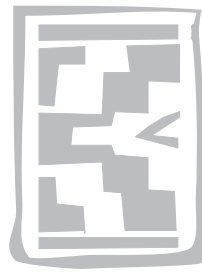

\title{
A check-list of the pentastomid parasites of crocodilians and freshwater chelonians
}

\author{
K. JUNKER and J. BOOMKER* \\ Department of Veterinary Tropical Diseases, University of Pretoria \\ Private Bag X04, Onderstepoort, 0110 South Africa
}

\begin{abstract}
JUNKER, K. \& BOOMKER, J. 2006. A check-list of the pentastomid parasites of crocodilians and freshwater chelonians. Onderstepoort Journal of Veterinary Research, 73:27-36

Based on published records and own data a summary is given of the geographical distribution of the currently known species of pentastomid parasites infecting crocodiles and alligators, as well as freshwater chelonians. A brief generic diagnosis is provided for each genus.
\end{abstract}

Fourteen out of the currently 23 living crocodilian species have been recorded as being host to one or more pentastomes. Out of the 32 pentastome species six are considered species inquirendae. Presently, six genera of crocodilian pentastomes, Agema, Alofia, Leiperia, Sebekia, Selfia and Subtriquetra are recognized. African crocodiles harbour eight pentastome species, six of which have been recorded from the Nile crocodile, Crocodylus niloticus. Three species belong to the genus Sebekia, Alofia being represented by two and Leiperia by only one species. Two species, Alofia parva and Agema silvaepalustris, occur in the dwarf crocodile, Osteolaemus tetraspis, and the slender-snouted crocodile, Crocodylus cataphractus, exclusively, but a single Sebekia species is shared with the Nile crocodile. The genus Agema is endemic to the African region. Infective stages of the pentastome Subtriquetra rileyi, thought to utilize Nile crocodiles as final hosts, have been recovered only from fishes. The largest number of pentastome species is found in the Australasian region. Of these, the Indo-Pacific crocodile, Crocodylus porosus, harbours seven, representing the genera Alofia, Sebekia, Leiperia and Selfia. Selfia is exclusive to the latter host. The genus Subtriquetra has been reported from "Indian crocodiles", a term possibly referring to either Crocodylus palustris, Crocodylus porosus or Gavialis gangeticus. Ten species of pentastomes parasitizing the crocodilian genera Alligator, Caiman, Crocodylus and Melanosuchus have been recorded from the Neotropical region including the southern states of the North American continent. The two most wide-spread pentastome genera, Alofia and Sebekia, have been recorded together with representatives of the genus Subtriquetra and immature and larval forms of Leiperia.

To date the two monospecific genera, Pelonia, from two terrapin species, Pelusios sinuatus and Pelomedusa subrufa, in South Africa, and Diesingia from Hydraspis geoffroyana and Hydromedusa tectifera in South America, are the only chelonian pentastomes recovered world-wide. A possible exception is the crocodilian pentastome Sebekia mississippiensis which can reach maturity in experimentally infected terrapins.

Keywords: Agema, Alligator, Alofia, Caiman, Crocodylus, Diesingia, Gavialis, Hydraspis, Hydromedusa, Leiperia, Melanosuchus, Pelomedusa, Pelonia, Pentastomida, Phrynops, Sebekia, Selfia, Subtriquetra, terrapins

* Author to whom correspondence is to be directed. E-mail: jboomker@op.up.ac.za

Accepted for publication 1 August 2005-Editor 


\section{INTRODUCTION}

The pentastomid parasites of chelonians and crocodilians are currently divided into the family Sebekidae and Subtriquetridae. The former comprises seven genera, namely, Agema, Alofia, Selfia, Sebekia, Leiperia, Diesingia and Pelonia. While the first four genera inhabit the lungs and bronchioles of the crocodilian host, Leiperia occurs in the trachea and bronchi (Riley, Spratt \& Winch 1990; Riley 1994; Riley \& Huchzermeyer 1996; Riley, Hill \& Huchzermeyer 1997). Diesingia and Pelonia parasitize the lungs of chelonian final hosts (Junker \& Boomker 2002; Junker, Riley \& Boomker 2003).

A single member of Sebekia, Sebekia mississippiensis, might be able to reach maturity in chelonians, too, but as yet no mature specimens have been collected from naturally infected hosts (Dukes, Shealy \& Rogers 1971). Members of the monogeneric family Subtriquetridae inhabit the nasopharynx of their crocodilian final hosts, but Subtriquetra rileyi, of which currently only infective larvae have been recovered, needs verification (Winch \& Riley 1986a; Junker, Boomker \& Booyse 1998).

During the past 10 years renewed progress has been made as regards the taxonomy of crocodilian pentastomes. The older genera, Alofia, Sebekia and Leiperia, have been revised, and examination of new material has led to the description of several new genera and species (Riley 1994; Riley et al. 1990, 1997; Riley \& Huchzermeyer 1996; Junker et al. 1998). However, there is a dearth of data concerning the chelonian pentastomids.

In order to provide a quick reference tool, this checklist consists of two parts, following the example of Sambon (1922). The first part lists the parasites under their scientific names, their synonyms and their authorities. A short generic diagnosis precedes each genus and the parasites are grouped according to the geographic distribution of their respective hosts. The list starts with Africa, followed by Australasia. South and North America are listed last.

The second part of the check-list alphabetically lists the hosts and their synonyms, and, also in alphabetical order, their respective parasites. The nomenclature and synonyms of the crocodilian and chelonian hosts are according to Getz (2002).

Only references dealing with mature pentastomes are included in the check-list, but for completeness' sake the intermediate hosts of pentastome species of which only larval forms are known are listed.

\section{PARASITE/HOST CHECK-LIST OF THE PENTASTOMIDA}

FAMILY SEBEKIDAE SAMBON, 1922

\section{Genus Agema Riley, Hill \& Huchzermeyer, 1997}

GENERIC DIAGNOSIS: Overall shape typical of smaller members of the Sebekidae; males claviform, females with more uniform diameter and conical posterior terminating in a small blunt point; adult hooks smooth; blades smoothly curved without abrupt right-angled bend near to base; blade on anterior hook pair larger than that of posterior pair; fulcrum without cowl; mouth ovoid and sides of cadre united anteriorly and posteriorly by segments of chitin which appear as two crescents; copulatory spicules delicate and elongate; basal section without a hooked collar (Riley et al. 1997).

\section{AfricA}

1. Agemasilvaepalustris Riley, Hill\& Huchzermeyer, 1997

Crocodylus cataphractus

Riley, Hill \& Huchzermeyer (1997), Republic of the Congo

Osteolaemus tetraspis

Riley, Hill \& Huchzermeyer (1997), Republic of the Congo

\section{Genus Alofia Giglioli, 1922}

GENERIC DIAGNOSIS: Size small; body banana-shaped; hooks large with long, narrow, slightly curved blade and a slender base; absence of chitinous formation at the base of the anterior hook; chitinous buccal cadre large and U-shaped; intestine as in Sebekia; found in crocodiles (Fain 1961 in Riley 1994); caudal extremity of female bluntly rounded, often swollen into a bulb (Riley 1994); hooks usually smooth (rarely with patches of minute spines [see Alofia nilotici and Alofia parva (Riley \& Huchzermeyer 1995a, b)], blades finely canaliculated, bent through almost a right angle at the base; peg-like extension of oral cadre projects into oesophagus; copulatory spicules with double-hooked collar on the shorter of the two anterior extensions (Riley \& Huchzermeyer 1995a, b; Junker, Boomker \& Bolton 1999).

\section{AfricA}

1. Alofia nilotici Riley \& Huchzermeyer, 1995 Crocodylus niloticus 
Riley \& Huchzermeyer (1995a), Botswana Junker, Boomker \& Bolton (1999), South Africa

2. Alofia parva Riley \& Huchzermeyer, 1995

Crocodylus cataphractus

Riley \& Huchzermeyer (1995b, 2000), Republic of the Congo

Osteolaemus tetraspis osborni

Riley \& Huchzermeyer (1995b, 2000), Republic of the Congo

3. Alofia simpsoni Riley, 1994

Unknown crocodilian

Riley (1994), Ghana

Crocodylus niloticus

Riley \& Huchzermeyer (1995a), Botswana

Junker, Boomker \& Bolton (1999), South Africa

\section{AUSTRALASIAN REGION}

4. Alofia ginae Giglioli, 1922

Unknown crocodilian

Sambon (1922), Samoa

Most probably Crocodylus porosus, as it is the only crocodilian whose range extends as far as Fiji in the Pacific Ocean and it is known to have colonized many small islands as far as nearly $1000 \mathrm{~km}$ from land (Ross 1989).

Crocodylus porosus (?)

Riley (1994), Philippines

The collector did not specify the host, but Riley (1994) concludes it to be C. porosus, as the only other Philippinian crocodile, Crocodylus mindorensis, does not occur in the region from which the parasites were recovered.

5. Alofia merki Giglioli, 1922

Sebekia merki Heymons, 1941

Unknown crocodilian

Sambon (1922), Samoa

Most probably Crocodylus porosus, as it is the only crocodilian whose range extends as far as Fiji in the Pacific Ocean and it is known to have colonized many small islands as far as nearly $1000 \mathrm{~km}$ from land (Ross 1989).

Crocodylus porosus

Riley (1994), Northern Territory, Australia

Riley (1994), Philippines

6. Alofia indica (Von Linstow, 1906) Hett, 1924, species inquirenda

\section{Gavialis gangeticus}

Hett (1924), India

\section{SOUTH \& NORTH AMERICAN REGION}

7. Alofia platycephala (Lohrmann, 1889) Giglioli, 1922

Pentastomum platycephalum Lohrmann, 1889; Porocephalus platycephalus Shipley, 1898; Reighardia platycephala Sambon, 1910

Unknown crocodilian

Lohrmann (1889), South America

Caiman crocodilus

Self \& Rego (1985), Brazil

Caiman latirostris

Heymons (1941), Paraguay

Comment: Hirst (1922) described Alofia adriatica (Hirst, 1922) Giglioli, 1922 from an unknown host from the Adriatic. As crocodilians do not occur in the Adriatic this species will have to remain species inquirenda until further material becomes available.

\section{Genus Leiperia Sambon, 1922}

GENERIC DIAGNOSIS: Female with spirally coiled abdomen; broad bands of chloride cells; hooks smooth, flat-topped with sharply curved blade; oral cadre Vshaped with large anterior flanges, numerous pores around the pharynx; copulatory spicules heavily chitinized with complex internal supporting structures, shorter of the two anterior extensions forms a smooth collar (double in L. australiensis) around the longer spatulate extension; cirrus tip modified into a flattened trumpet of longitudinally-striated chitin (Riley \& Huchzermeyer 1996; Junker, Boomker, Swanepoel \& Taraschewski 2000).

AFRICA

1. Leiperia cincinnalis (Vaney \& Sambon, 1910) Sambon, 1922

Reighardia cincinnalis Vaney \& Sambon, 1910; 
check-list of pentastomid parasites of crocodilians and freshwater chelonians

Porocephalus nematoides De Beauchamp, 1918 Crocodylus cataphractus

Fain (1961), Central Africa: infective larva Crocodylus niloticus

Vaney \& Sambon (1910), Uganda

Sambon (1922), Zimbabwe

Rodhain \& Vuylsteke (1932), Democratic Republic of the Congo

Junker,Boomker, Swanepoel\& Taraschewski (2000), South Africa

\section{AUSTRALASIAN REGION}

2. Leiperia australiensis Riley \& Huchzermeyer, 1996

Crocodylus johnsoni

Riley \& Huchzermeyer (1996), Northern

Territory, Australia

Crocodylus porosus

Riley \& Huchzermeyer (1996), Northern Territory, Australia

\section{SOUTH \& NORTH AMERICAN REGION}

3. Leiperia gracilis Diesing, 1936, species inquirenda

Pentastoma gracile Diesing, 1836 (partim); Pentastomum gracile Leidy, 1856; Pentastoma gracilis Parona, 1891; Porocephalus gracilis Shipley, 1898; Porocephalus crocodiliWheeler, 1915 (partim); Leiperia neotropica Heymons \& Vitzthum, 1935

Immature and larval forms were recovered from the following hosts, but adults have not been collected.

Alligator mississippiensis

Leidy (1856, in Sambon 1922), locality unknown, North America

Crocodylus acutus

Heymons (1935), South America

Caiman crocodilus

Heymons (1935), Brazil

\section{Genus Sebekia Sambon, 1922}

GENERIC DIAGNOSIS: Body short and squat with 5894 compressed annuli; lateral lines conspicuous; cephalothorax small, wedge-shaped and projecting nipple-like from the abdomen, ventral surface continuous with that of the abdomen; mouth subterminal and shaped like an inverted ' $U$ '; oral cadre oval to elongate, highly variable in shape, without long, parallel sides and generally united anteriorly; hooks small, equal or subequal, claw-shaped, with convex or flat dorsal surface; all hooks spiny (rarely only the anterior pair); all fulcra often with spinous anterior extension (rarely only the anterior pair); hook barb curved, strongly united and continuous with shank; spicules generally obpyriform, with one or two fine sclerotized rods supporting membranous region distally. Parasites of the lungs of crocodilians, rarely of chelonians (Riley et al. 1990).

AFRICA

1. Sebekia cesarisi Giglioli, 1922

Crocodylus sp.

Sambon (1922), Africa

Crocodylus niloticus

Riley \& Huchzermeyer (1995a), Botswana

Junker, Boomker \& Bolton (1999), South Africa

2. Sebekia okavangoensis Riley \& Huchzermeyer, 1995

Sebekia cesarisi Riley, Spratt \& Winch, 1990

Crocodylus cataphractus

Riley \& Huchzermeyer (2000), Republic of the Congo

Crocodylus niloticus

Riley \& Huchzermeyer (1995a), Botswana Junker, Boomker \& Bolton (1999), South Africa

Osteolaemus tetraspis

Riley \& Huchzermeyer (2000), Republic of the Congo

3. Sebekia wedli Giglioli, 1922

Pentastoma oxycephalum var. minor Wedli, 1861; Sebekia oxycephala Self \& Rego, 1985

Crocodylus niloticus

Devos (1939), Democratic Republic of the Congo

Riley, Spratt \& Winch (1990), Uganda

Riley \& Huchzermeyer (1995a), Botswana

Junker, Boomker \& Bolton (1999), South Africa 
AustRALASIAN REGION

4. Sebekia johnstoni Riley, Spratt \& Winch, 1990

Crocodylus johnsoni

Riley, Spratt \& Winch (1990), Northern Territory, Australia

Crocodylus porosus

Riley, Spratt \& Winch (1990), Northern Territory, Australia

5. Sebekia multiannulata Riley, Spratt \& Winch, 1990

Crocodylus johnsoni

Riley, Spratt \& Winch (1990), Northern

Territory, Australia

Crocodylus porosus

Riley, Spratt \& Winch (1990), Northern Territory, Australia

6. Sebekia purdieae Riley, Spratt \& Winch, 1990

Crocodylus porosus

Riley, Spratt \& Winch (1990), Northern Territory, Australia

7. Sebekia jubini (Vaney \& Sambon, 1910) Sambon, 1922, species inquirenda

Porocephalus jubini Vaney \& Sambon, 1910

Crocodylus siamensis

Sambon (1922), locality unknown, southeast Asia

8. Sebekia novaeguineae Riley, Spratt \& Winch, 1990

Crocodylus novaeguineae

Riley, Spratt \& Winch (1990), Papua New Guinea

\section{SOUTH \& NORTH AMERICAN REGION}

9. Sebekia acuminata Travassos, 1924, species inquirenda

Unknown crocodilian

Travassos (1924), Brazil

10. Sebekia divestei Giglioli, 1922

Crocodylus acutus

Sambon (1922), locality unknown, Neotropical region

11. Sebekia microhamus Self \& Rego, 1985
Caiman crocodilus

Self \& Rego (1985), Brazil

12. Sebekia mississippiensis Overstreet, Self \& Vliet, 1985

Pentastoma oxycephalum Diesing, 1836 (partim); Pentastomum gracile (syn. Leiperia gracilis) Leidy, 1856

Alligator mississippiensis

Deakins (1971), USA

Hazen, Aho, Murphy, Esch \& Schmidt (1978), USA

Overstreet, Self \& Vliet (1985), USA

13. Sebekia oxycephala (Diesing, 1836) Sambon, 1922

Pentastoma proboscideum Rudolphi, 1819 (partim); Pentastoma oxycephalum Diesing, 1836 (partim); Pentastoma gracile Diesing, 1836 (partim); Pentastomum oxycephalum Diesing, 1850 (partim); Pentastomum gracile Diesing, 1850 (partim); Pentastomum heterodontis Leuckart, 1860; Pentastomum oxycephalum Chatin, 1882; Porocephalus oxycephalus Stiles, 1893; Pentastoma proboscideum crocodili scleropis Rudolphi (Shipley in Sambon 1922); Reighardia oxycephala Vaney \& Sambon, 1910; Porocephalus crocodili Wheeler, 1913 (partim); Sebekia oxycephala Sambon, 1922 (partim); Bdukus ichthyius Holl, 1929; Leiperia heterodontis Heymons \& Vitzthum, 1935; Sebekia crocodili Heymons \& Vitzthum, 1935

Alligator mississippiensis

Sambon (1922), locality unknown

Caiman crocodilus

Sambon (1922), locality unknown

Winch \& Riley (1986b), Trinidad, South America

Caiman latirostris

Heymons (1941), locality unknown

Crocodylus acutus

Sambon (1922), locality unknown

14. Sebekia samboni Travassos, 1924, species inquirenda

Unknown crocodilian

Travassos (1924), Brazil

15. Sebekia trinitatis Riley, Spratt \& Winch, 1990 
check-list of pentastomid parasites of crocodilians and freshwater chelonians

\section{Caiman crocodilus}

Riley, Spratt \& Winch (1990), Trinidad, South America

\section{Genus Selfia Riley, 1994}

GENERIC DIAGNOSIS: Size small, cephalothorax minute in comparison with diameter of the abdomen; 78-82 well defined annuli; abdomen strongly curled ventrally; caudal extremity of female abruptly tapered to blunt point; hooks very small, with tiny blade only slightly offset from transversely creased and folded shank; rear of anterior hooks enveloped by soft, spinous cowl which forms an extension of the fulcrum; buccal cadre somewhat variable in shape, being oval to more U-shaped, but lacking parallel sides; copulatory spicule of male like that of Alofia (Riley 1994).

\section{AUSTRALASIAN REGION}

1. Selfia porosus Riley, 1994

Crocodylus porosus

Riley (1994), Northern Territory, Australia

\section{Genus Diesingia Sambon, 1922}

GENERIC DIAGNOSIS: Hooks smooth, flat-topped, with sharply curved blades; fulcra with anterior cowl-like extension, extension smooth in posterior and spiny in anterior fulcra; oral cadre open anteriorly with an oesphageal peg similar to that in Alofia; copulatory spicule with cowry shell-shaped base, the short, ventral extension is transformed into a structure resembling the collembolan fulcrum, and is connected to the base by a joint (Junker, Riley \& Boomker 2003).

\section{SOUTH \& NORTH AMERICAN REGION}

1. Diesingia megastoma (Diesing, 1836) Sambon, 1922

Pentastoma megastomum Diesing, 1836; Pentastomum megastomum Leuckart, 1860; Porocephalus megastomus Shipley, 1898; Sebekia megastoma Travassos, 1923; Sebekia crocodili Heymons \& Vitzthum, 1935; Diesingia megastoma Heymons; 1941; Butantanella megastoma Da Fonseca \& Ruiz, 1956; Sebekia megastoma Self \& Rego, 1985

Phrynops geoffroanus

Diesing (1836), Brazil

Hydromedusa tectifera

Da Fonseca \& Ruiz (1956), Brazil

\section{Genus Pelonia Junker \& Boomker, 2002}

GENERIC DIAGNOSIS: Hooks smooth, dorsally convex, with sharply bent blades, fulcra without extensions; oral cadre more or less U-shaped, closed anteriorly by delicate chitinous fibres; copulatory spicules almost identical to those of Sebekia wedli, with cowry shell-shaped base and the short anterior extension ending in a smooth collar, the long spatulate extension carries small chitinous teeth (Junker \& Boomker 2002).

AFRICA

1. Pelonia africana Junker \& Boomker, 2002

Pelomedusa subrufa Junker \& Boomker (2002), South Africa

Pelusios sinuatus Junker \& Boomker (2002), South Africa

FAMILY SUBTRIQUETRIDAE FAIN, 1961

\section{Genus Subtriquetra Sambon, 1922}

GENERIC DIAGNOSIS: Body elliptical, ventrally flattened and dorsally dome shaped with flattened margins; hooks simple, slender and sharply pointed, disposed in a curved line; oral cadre rounded (Fain 1961; Winch \& Riley 1986a; Junker et al. 1998).

AFRICA

1. Subtriquetra rileyi Junker, Boomker \& Booyse, 1998

Infective larvae:

Tilapia rendalli swierstrae

Junker, Boomker \& Booyse (1998), South Africa

Oreochromis mossambicus

Junker, Boomker \& Booyse (1998), South Africa

AUSTRALASIAN REGION

2. Subtriquetra megacephala (Baird, 1853) Sambon, 1922

Pentastoma megacephalum Baird, 1853; Porocephalus megacephalus Shipley, 1898

Crocodylus palustris

Sambon (1922), Sunderbunds, India

Crocodylus palustris, Crocodylus porosus or 
Gavialis gangeticus? ("Sangor crocodile")

Sambon (1922), Bengal, India

3. Subtriquetra shipleyi Hett, 1924

Crocodylus palustris Crocodylus porosus or Gavialis gangeticus? ("Indian crocodile")

Hett (1924), India

SOUTH \& NORTH AMERICAN REGION

4. Subtriquetra subtriquetra (Diesing, 1836)

Pentastoma proboscideum Bresmer, 1824 (partim); Pentastoma subtriquetrum Diesing, 1836; Pentastomum subtriquetrum Diesing, 1850; Pentastomum pusillum Diesing, 1856; Linguatula subtriquetra Raillet, 1883; Linguatula pusilla Shipley, 1898

Caiman crocodilus

Sambon (1922), South America

Winch \& Riley (1986a), Trinidad, South America

Melanosuchus niger

Sambon (1922), South America

\section{HOST/PARASITE CHECK-LIST OF THE PENTASTOMIDA}

\section{Crocodylia}

FAMILY ALLIGATORIDAE (CUVIER, 1807)

(Alligators and caimans)

\section{Genus Alligator Cuvier, 1807}

1. Alligator mississippiensis (Daudin, 1801) Daudin, 1802 (American alligator)

Crocodilus mississipiensis Daudin, 1801

Leiperia gracilis, species inquirenda, larval forms only

Sebekia mississippiensis

Sebekia oxycephala

\section{Genus Caiman Spix, 1825}

1. Caiman crocodilus (Linnaeus, 1758) (Common or Spectacled caiman)

Lacerta crocodilus Linnaeus, 1758; Caiman sclerops Schneider, 1801 (fide Medem 1981); Perosuchus fuscus Cope, 1868; Alligator (Jacare) chiapasius Bocourt, 1876

Alofia platycephala

Leiperia gracilis, species inquirenda, larval forms

Sebekia microhamus

Sebekia oxycephala

Sebekia trinitatis

Subtriquetra subtriquetra

2. Caiman latirostris (Daudin, 1801) (Broad-snouted caiman)

Crocodilus latirostris Daudin, 1801; Caiman fissipes Spix, 1825; Champsa fissipes Wagler, 1828 (fide Hoogmoed \& Gruber, 1983); Alligator cynocephalus Duméril \& Bibron, 1836; Jacare latirostris Gray, 1862; Alligatorlatirostris Boulanger, 1886; Jacaretinga latirostris Vaillant, 1898

Alofia platycephala

Sebekia oxycephala

\section{Genus Melanosuchus Gray, 1862}

1. Melanosuchus niger (Spix, 1825) (Black caiman) Caiman niger Spix, 1825

Subtriquetra subtriquetra

FAMILY CROCODYLIDAE (CUVIER, 1807) (Crocodiles)

Subfamily Crocodylinae (Cuvier, 1807)

\section{Genus Crocodylus Laurenti, 1768}

1. Crocodylus acutus (Cuvier, 1807) (American crocodile)

Crocodilus acutus Cuvier, 1807

Leiperia gracilis, species inquirenda, larval forms only

Sebekia divestei

Sebekia oxycephala

2. Crocodylus cataphractus Cuvier, 1825 (Slendersnouted crocodile)

Crocodilus cataphractus Falconer, 1846

Agema silvaepalustris

Alofia parva

Leiperia cincinnalis, infective larva

Sebekia okavangoensis 
check-list of pentastomid parasites of crocodilians and freshwater chelonians

3. Crocodylus johnsoni Krefft, 1873 (Australian freshwater crocodile)

Tomistoma kreffti Gray in Krefft, 1873 (nomen nudum); Crocodilus (Philas) johnstoni Gray, 1874; Crocodylus johnstoni Cogger, 2000

Leiperia australiensis

Sebekia johnstoni

Sebekia multiannulata

4. Crocodylus niloticus Laurenti, 1768 (Nile crocodile)

Crocodilus vulgaris Cuvier, 1807; Crocodilus multiscutatus Rüppell in Cretzschmar, 1826; Crocodilus marginatus Geoffroy, 1827; Crocodilus madagascariensis Grandidier, 1872; Crocodilus vulgaris var. madagascariensis Boettger, 1877

Alofia nilotici

Alofia simpsoni

Leiperia cincinnalis

Sebekia cesarisi

Sebekia okavangoensis

Sebekia wedli

5. Crocodylus novaeguineae Schmidt, 1928 (New Guinea crocodile)

Sebekia novaeguineae

6. Crocodylus palustris Lesson, 1831 (Mugger, Marsh crocodile)

Subtriquetra megacephala

Subtriquetra shipleyi ("Indian crocodile")

7. Crocodylus porosus Schneider, 1801 (IndoPacific or Saltwater crocodile)

Crocodylus natans Meyer, 1795; Crocodilus porosus Schneider, 1801; Crocodilus oopholis Schneider, 1801; Crocodilus biporcatus Cuvier, 1807; Crocodilus biporcatus raninus Müller \& Schlegel, 1844; Oopholis pondicherianus Gray, 1862; Crocodylus porosus australis Deraniyagala, 1953; Crocodylus porosus minikanna Deraniyagala, 1953

Alofia ginae (possibly Crocodylus mindorensis, but distributionally unlikely)

Alofia merki

Leiperia australiensis

Sebekia johnstoni

Sebekia multiannulata

Sebekia purdieae
Selfia porosus

Subtriquetra shipleyi ("Indian crocodile")

8. Crocodylus siamensis Schneider, 1801 (Siamese crocodile)

Crocodilus galeatus Cuvier, 1807

Sebekia jubini, species inquirenda

\section{Genus Osteolaemus Cope, 1861}

1. Osteolaemus tetraspis Cope, 1861 (Dwarf crocodile)

Crocodilus frontatus Murray, 1862; Halcrosia afzelii Lilljeborg, 1867; Halcrosia nigra Gray, 1867; Halcrosia nigra Gray, 1870; Osteoblepharon osborni Schmidt, 1919; Osteolaemus tetraspis tetraspis Wermuth \& Mertens, 1961

Agema silvaepalustris

Alofia parva

Sebekia okavangoensis

FAMILY GAVIALIDAE ADAMS, 1854 (Gharials)

\section{Genus Gavialis Oppel, 1811}

1. Gavialis gangeticus (Gmelin, 1789) (Gharial)

Lacerta gangetica Gmelin, 1789

Subtriquetra megacephala ("Indian crocodile")

Subtriquetra shipleyi ("Indian crocodile")

\section{CHELONIA}

\section{Suborder Pleurodira (Side-necked turtles)}

FAMILY PELOMEDUSIDAE COPE, 1868

\section{Genus Pelomedusa Wagler, 1830}

1. Pelomedusa subrufa (Lacépède, 1788) (Cape terrapin)

Testudo subrufa Lacépède, 1788; Testudo galeata Schoepff, 1792; Testudo badia Donndorf, 1798; Testudo rubicunda Suckow, 1798; Emys olivacea Schweigger, 1812 (non Emys olivacea Gray, 1855); Pentonyx capensis Duméril \& Bibron, 1835; Pentonyx gehafie Rüppell, 1835; Pentonix americana Cornalia, 1849; Pelomedusa mozambica Peters (nomen nudum) in Gray 1855 (?); Pelomedusa mossambicensis Peters (nomen nudum) in Lichtenstein 1856; Pelomedusa 
nigra Gray, 1863; Pelomedusa gasconi Rochebrune, 1884; Pelomedusa galeata Boulanger, 1889; Pelomedusa galeata var. disjuncta Vaillant \& Grandidier, 1910; Pelomedusa galeata orangensis Hewitt, 1935; Pelomedusa galeata devilliersi Hewitt, 1935; Pelomedusa galeata damarensis Hewitt, 1935; Pelomedusa subrufa wettsteini Mertens, 1937; Testudo emys arabica N.-Ehrenberg in Stresemann 1954

Pelonia africana

\section{Genus Pelusios Wagler, 1830}

1. Pelusios sinuatus (Smith, 1838) (Serrated hinged terrapin, African serrated mud turtle)

Sternotherus sinuatus Smith, 1838; Sternotherus dentatus Peters, 1848 (nomen nudum); Sternothaerus sinuatus Boulanger, 1889; Sternothaerus bottegi Boulanger, 1895; Pelusios sinuatus zuluensis Hewitt, 1927; Pelusios sinuatus leptus Hewitt, 1927

Pelonia africana

FAMILY CHELIDAE GRAY, 1825 (Snake-necked turtles)

\section{Genus Hydromedusa Wagler, 1830}

1. Hydromedusa tectifera Cope, 1869 [1870] (South American snake-necked turtle, Uruguay snakenecked turtle)

Hydromedusa platanensis Gray, 1873; Hydromedusa wagleri Günther, 1884

\section{Diesingia megastoma}

\section{Genus Phrynops Wagler, 1830}

1. Phrynops geoffroanus (Schweigger, 1812) Gorzula \& Señaris, 1999 (Geoffroy's side-necked turtle)

Emys geoffroana Schweigger, 1812; Emys geoffreana Schweigger, 1812 (fide Boulanger, 1886); Emys depressa Merrem, 1820 (non Emys depressa Spix, 1824); Emys viridis Spix, 1824 (?); Emys geoffroyana Gray, 1831; Platemys geoffreana Duméril \& Bibron, 1835; Platemys neuwiedii Duméril \& Bibron, 1835; Platemys waglerii Duméril \& Bibron, 1835; Platemys tuberosa Peters, 1870; Platemys geoffroyana Boulanger, 1886; Hydraspis geoffroyana Boulanger, 1889; Hydraspis wagleri Boulanger, 1889; Hydraspis tuberosa Boulanger, 1889; Hydraspis boulangeri Bohls,
1895; Phrynops geoffroana Mertens et al., 1934; Phrynops geoffroana geoffroana Müller, 1939; Phrynops tuberosa Mertens etal., 1934; Phrynops geoffroana tuberosa Müller, 1939

\section{Diesingia megastoma}

\section{REFERENCES}

DA FONSECA, F. \& RUIZ, J.M. 1956. Was ist eigentlich Pentastoma megastomum Diesing, 1836? (Porocephalida, Porocephalidae). Senckenbergiana Biologica, 37:469-485.

DEAKINS, D.E. 1971. Pentastomes from Blackbeard Island, Georgia, with notes on North American pentastomes. Journal of Parasitology, 57:1197.

DEVOS, R. 1939. Deux porocephales des crocodiles de Luapula. Annales de la Société Belge de Médecine Tropicale, 19:193195.

DIESING, K.M. 1836. Versuch der Monographie der Gattung Pentastoma. Annalen des Wiener Museums der Naturgeschichte, 1:1-32.

DUKES, G.H., SHEALY, R.M. \& ROGERS, W.A. 1971. Sebekia oxycephala (Pentastomida) in largemouth bass from Lake St. John, Concordia Parish, Louisiana. Journal of Parasitology, 57:1028.

FAIN, A. 1961. Pentastomides de l' Afrique Centrale. Annales du Musée Royale de l' Afrique Centrale, Série 8, 92:1-115.

GETZ, W. 2002. Reptilia. http://srs.embl-heidelberg.de:8000/

HAZEN, T.C., AHO, J.M., MURPHY, T.M., ESCH, G.W. \& SCHMIDT, G.D. 1978. The parasite fauna of the American alligator (Alligator mississippiensis) in South Carolina. Journal of Wildlife Diseases, 14:435-439.

HETT, M.L. 1924. On the family Linguatulidae. Proceedings of the Zoological Society of London, 1924: 107-159.

HEYMONS, R. 1935. Pentastomida, in Bronns Klassen und Ordnungen des Tierreichs, Vol. 5. Leipzig: Akademische Verlagsgesellschaft.

HEYMONS, R. 1941. Beiträge zur Systematik der Pentastomiden VI-Die Arten der Gattung Alofia im Vergleich mit Sebekia. Zeitschrift für Parasitenkunde, 12:419-432.

HIRST, S. 1922. On a new linguatulid from the Adriatic. Annals and Magazine of Natural History, 10:289.

JUNKER, K. \& BOOMKER, J. 2002. Description of Pelonia africana n. g., n. sp. (Pentastomida: Sebekidae) from the lungs of Pelomedusa subrufa and Pelusios sinuatus (Chelonia) in South Africa. Onderstepoort Journal of Veterinary Research, 69:53-59.

JUNKER, K., BOOMKER, J. \& BOLTON, L. 1999. Pentastomid infections in the Nile crocodile (Crocodylus niloticus) in the Kruger National Park, South Africa, with a description of the males of Alofia simpsoni. Onderstepoort Journal of Veterinary Research, 66:65-71.

JUNKER, K., BOOMKER, J. \& BOOYSE, D.G. 1998. Pentastomid infections in cichlid fishes in the Kruger National Park and the description of the infective larva of Subtriquetra rileyi n. sp.. Onderstepoort Journal of Veterinary Research, 65:159-167.

JUNKER, K., BOOMKER, J., SWANEPOEL, D. \& TARASCHEWSKI, H. 2000. Leiperia cincinnalis Sambon, 1922 from Nile crocodiles Crocodylus niloticus in the Kruger National Park, South Africa, with a description of the male. Systematic Parasitology, 47:29-41. 
JUNKER, K., RILEY, J. \& BOOMKER, J. 2003. Redescription of Diesingia megastoma (Diesing, 1836) Sambon, 1922, a pentastomid parasite from the South American terrapin Hydromedusa tectifera. Systematic Parasitology, 56:211-218.

LOHRMANN, E. 1889. Untersuchungen über den anatomischen Bau der Pentastomen. Archiv für Naturgeschichte, 1:303337.

OVERSTREET, R.M., SELF, J.T. \& VLIET, K.A. 1985. The pentastomid Sebekia mississippiensis sp. $\mathrm{n}$. in the American alligator and other hosts. Proceedings of the Helminthological Society of Washington, 52:266-277.

RILEY, J. 1994. A revision of the genus Alofia Giglioli, 1922 and a description of a new monotypic genus, Selfia: two genera of pentastomid parasites (Porocephalida: Sebekidae) inhabiting the bronchioles of the marine crocodile Crocodylus porosus and other crocodilians. Systematic Parasitology, 29: 23-41.

RILEY, J., HILL, G.F. \& HUCHZERMEYER, F.W. 1997. A description of Agema, a new monotypic pentastomid genus from the lungs of the African dwarf and slender-snouted crocodiles. Systematic Parasitology, 37:207-217.

RILEY, J. \& HUCHZERMEYER, F.W. 1995a. Description of four species of pentastomid parasites belonging to the genera Alofia Giglioli, 1922 and Sebekia Sambon, 1922, from a single Nile crocodile Cocodylus niloticus from Botswana. Systematic Parasitology, 31:221-238.

RILEY, J. \& HUCHZERMEYER, F.W. 1995b. Pentastomid parasites of the family Sebekidae Fain, 1961 in West African dwarf crocodiles Osteolaemus tetraspis Cope, 1851 from the Congo, with a description of Alofia parvan. sp. Onderstepoort Journal of Veterinary Research, 62:151-162.

RILEY, J. \& HUCHZERMEYER, F.W. 1996. A reassessment of the pentastomid genus Leiperia Sambon, 1922 with a description of a new species from both the Indopacific crocodile Crocodylus porosus and Johnston's crocodile $C$. johnstoni in Australia. Systematic Parasitology, 34:53-66.
RILEY, J. \& HUCHZERMEYER, F.W. 2000. Diet and lung parasites of Swamp forest dwarf crocodiles (Osteolaemus tetraspis osborni) in the Northern Congo Republic. Copeia, 2: 582-586.

RILEY, J., SPRATT, D.M. \& WINCH, J.M. 1990. A revision of the genus Sebekia Sambon, 1922 (Pentastomida) from crocodilians with descriptions of five new species. Systematic Parasitology, 16:1-25.

RODHAIN, J. \& VUYLSTEKE, C. 1932. Contribution à I' étude des porocéphales des crocodiles africains. Révue de Zoologie et de Botanique Africaines, 23:1-11.

ROSS, C.A (Ed.). 1989. Crocodiles and alligators, London: Meerehurst Press.

SAMBON, L.W. 1922. A synopsis of the family Linguatulidae. Journal of Tropical Medicine and Hygiene, 25:188-206; 391-428.

SELF, J.T. \& REGO, A.A. 1985. Reassessments and revisions of certain genera and species of the family Sebekidae (Pentastomida) including a description of Sebekia microhamus $\mathrm{n}$. sp.. Systematic Parasitology, 7:33-41.

TRAVASSOS, L. 1924. Sebekia du poumon des crocodiles d'Amerique. Comptes rendus hebdomaires des séances et mémoires de la Société de Biologie, 90:239-240.

VANEY, C. \& SAMBON, L.W. 1910. Preliminary notes on three new species of tongue worms (Linguatulidae) in the collection of the "Museum d'Histoire Naturelle", Paris. Transactions of the Royal Society of Tropical Medicine and Hygiene, 3: $129-134$.

WINCH, J.M. \& RILEY, J. 1986a. Studies on the behaviour, and development in fish, of Subtriquetra subtriquetra: a uniquely free-living pentastomid larva from a crocodilian. Parasitology, 93:81-98.

WINCH, J.M. \& RILEY, J. 1986b. Morphogenesis of larval Sebekia oxycephala (Pentastomida) from a South American crocodilian (Caiman sclerops) in experimentally infected fish. Zeitschrift für Parasitenkunde, 72:251-264. 